\title{
Motivations and Key Features for a Wearable Device for Continuous Monitoring of Breathing: A Web-Based Survey
}

Joseph Barry Prinable ${ }^{1,2}$, B Eng (Hons); Juliet M Foster ${ }^{2}$, PhD; Alistair L McEwan ${ }^{1}$, BE, PhD; Paul M Young ${ }^{2}$, PhD; Euan Tovey ${ }^{2}, \mathrm{PhD}$; Cindy Thamrin ${ }^{2}, \mathrm{PhD}$

${ }^{1}$ Faculty of Engineering and Information Technologies, School of Electrical and Information Engineering, University of Sydney, Sydney, Australia

${ }^{2}$ Woolcock Institute of Medical Research, University of Sydney, Glebe, Australia

\section{Corresponding Author:}

Joseph Barry Prinable, B Eng (Hons)

Faculty of Engineering and Information Technologies

School of Electrical and Information Engineering

University of Sydney

Rm 340, J03

Sydney, 2006

Australia

Phone: 61293517256

Email: jpri6895@uni.sydney.edu.au

\section{Abstract}

Background: Analysis of patterns of breathing over time may provide novel information on respiratory function and dysfunction. Devices that continuously record and analyze breathing rates may provide new options for the management of respiratory diseases. However, there is a lack of information about design characteristics that would make such devices user-friendly and suitable for this purpose.

Objective: Our aim was to determine key device attributes and user requirements for a wearable device to be used for long-term monitoring of breathing.

Methods: An online survey was conducted between June and July 2016. Participants were predominantly recruited via the Woolcock Institute of Medical Research database of volunteers, as well as staff and students. Information regarding the survey, a consent form, and a link to a Web-based questionnaire were sent to participants via email. All participants received an identical survey; those with doctor-diagnosed asthma completed an extra questionnaire on asthma control (Asthma Control Test). Survey responses were examined as a group using descriptive statistics. Responses were compared between those with and without asthma using the chi-square test.

Results: The survey was completed by 134 participants (males: 39\%, median age group: 50-59 years, asthma: 57\%). Of those who completed the Asthma Control Test, 61\% (47/77) had suboptimal asthma control. Of the 134 participants, 61.9\% (83/134) would be willing to wear a device to monitor their breathing, in contrast to $6.7 \%(9 / 134)$ who would not. The remaining $31.3 \%$ (42/134) stated that their willingness depended on specific factors. Participants with asthma most commonly cited their asthma as motivation for using a wearable; the most common motivation for use in those without asthma was curiosity. More than $90 \%$ of total participants would use the device during the day, night, or both day and night. Design preferences among all users included a wrist watch (nominated by $92.5 \%$ [124/134] for both day and night use, out of four body sites), the ability to synchronize breathing data with a mobile phone or tablet $(81.3 \%, 109 / 134)$, overnight power charging $(33.6 \%, 45 / 134)$, and a cost of $\leq$ Aus $\$ 100(53.7 \%, 72 / 134)$.

Conclusions: We have explored the motivations and likelihood for adopting wearable technologies for the purpose of monitoring breathing and identified user preferences for key design features. We found participants were motivated to adopt a wearable breathing monitor irrespective of health status, though rationale for use differed between those with and without asthma. These findings will help inform the design of a user-acceptable wearable device that will facilitate its eventual uptake in both healthy and asthma populations.

(JMIR Biomed Eng 2017;2(1):e1) doi: 10.2196/biomedeng.7143 


\section{KEYWORDS}

asthma; wearable; breathing; e-health

\section{Introduction}

Asthma is a serious public health problem affecting over 300 million people globally. Management challenges include the early prediction or warning of asthma attacks and optimizing the pharmaceutical management of the disease.

Monitoring of lung function over time is a widely accepted component of the assessment of asthma, both in clinical management of the disease as well as in research trials [1]. Some studies suggest it may also yield insights into the pathology of respiratory diseases and predict future risk of exacerbations [2-4]. In asthma, monitoring is usually based on standard lung function testing involving forced breathing maneuvers assessed periodically in a specialized respiratory laboratory, or by peak expiratory flow measured in a general practice and then in the patient's home either daily or during periods of worsening symptoms. There is a paucity of research on continuous, real-time monitoring of breathing for general health or for management of asthma or other chronic diseases. This may be due in part to the lack of commercial technology to enable such monitoring in a manner that would be acceptable to users. One study has shown that monitoring respiratory rate could help predict the onset of exacerbations in chronic obstructive pulmonary disease [5]. However, it is not known whether monitoring of breathing could aid diagnosis or monitoring of asthma. Breathing monitoring may also provide rapid feedback to a patient during physical exertion or breathing exercises during exacerbation episodes.

Several studies have investigated desirable features for a wearable device for health monitoring, from both a technical [6] and human-centered [7-11] perspective. These studies have provided guidelines on wearable design [7-9] and determined that user acceptability was dependent on factors such as fundamental needs/demonstrated benefit, enjoyment, and social value $[10,11]$. However, none of these studies sought to specifically determine the desired features for a wearable device used for long-term respiratory monitoring. At present, there are several modalities and locations on the body identified for respiratory monitoring: the ear, throat region, finger, wrist, and chest [12-14]. In the design and development of a device for this purpose, it is important to first identify, understand, and consider user preferences to increase user acceptance, satisfaction, and engagement $[8,15]$.

The purpose of this study is to (1) explore the reasons why participants with or without asthma would potentially adopt new technologies to monitor breathing over time, and (2) evaluate device-specific attributes that would meet the expectation of users within these two groups. We chose to additionally study healthy individuals, not only as a basis for comparison with those with asthma to identify those preferences that are specific to asthma, but also due to the increasing interest in personal health monitoring in the general population as evidenced by the uptake of wearable devices that measure activity and other physiological life signs.

\section{Methods}

\section{Study Design and Overview}

An online survey was conducted between June and July 2016. A link to the survey was sent electronically to a subset $(n=569)$ of the Woolcock Institute of Medical Research Volunteers Database based on the availability of a valid email address on record, as well as to staff and students at the Woolcock Institute. During the recruitment period, two rounds of recruitment emails were sent to the two lists, followed by a subsequent reminder email for each round. The Volunteers Database consists of members of the public who have previously given consent to be contacted about participation in research. The database comprises both healthy individuals $(n=256)$ as well as those with asthma $(n=1173)$. The exact number reached may differ due to constant additions or withdrawals from the database and the possibility of family members sharing a common email address. Inclusion criteria were (1) provision of informed consent, (2) completion of all responses, (3) no respiratory illness reported (for the healthy group), and (4) self-reported doctor's diagnosis of asthma (for the asthma group). No incentives were offered for participation. The protocol for this study was approved by Northern Sydney Local Health District Human Research Ethics Committee (ethics approval \#LNR/16/HAWKE99).

\section{Survey}

After clicking on the link to the survey, participants who provided informed consent proceeded to fill out an online questionnaire (see Multimedia Appendix 1) that took approximately 10-15 minutes to complete. The survey was designed to assess participant's current use of technology, to explore their readiness to use a wearable, and to understand their attitude toward the potential usefulness of wearable technologies for monitoring breathing. Specifically, the survey aimed to identify usage preferences (eg, how long the user wishes to wear the device during the night/day) and feature preferences, such as the device form factor (eg, band, sticky patch, earpiece), body location (eg, wearable for neck, chest, ear, wrist), display, charge time, and price.

The survey also included demographic questions such as age, gender, educational and socioeconomic status, and doctor-diagnosed health conditions. Those who reported having a doctor diagnosis of asthma completed the Asthma Control Test (ACT) [16], a well-validated scale [17], which comprises five questions that assess asthma symptoms, use of medication, and the effect of asthma on daily functioning to determine overall asthma control status. The total score ranges from 5 (poor control of asthma) to 25 (complete control of asthma); a score of $\leq 19$ indicates suboptimal control.

\section{Statistical Analyses}

Participant demographics were summarized using descriptive statistics. Results were compared between participants with self-reported doctor-diagnosed asthma versus those without 
asthma, using $t$ tests or Wilcoxon signed rank sum test depending on whether the data were normally distributed. Participants who were "unsure" of their asthma status were grouped with those participants without asthma. Questionnaire responses were compared between asthma and no asthma, between gender, and between age groups using chi-square tests. Statistical analyses were performed using SPSS v. 23 (IBM Corp.), and graphs were generated using Prism v. 7 (GraphPad Software Inc.).

\section{Results}

\section{Demographics}

In total, 156 participants responded but 2 did not provide informed consent and 20 failed to complete more than $50 \%$ of the survey and were omitted from analysis. Of the 134 participants who completed the survey (ie, $85.9 \%$ completion rate), 131 provided demographic information as shown in Table 1. Just under a third $(29.1 \%, 39 / 134)$ of participants were male, and nearly two-thirds $(60.0 \%, 79 / 134)$ had a university education. More than 10 participants were obtained in each age group. The average time to complete the survey was 13 minutes.

A total of $61.2 \%(76 / 134)$ participants reported doctor-diagnosed asthma: mean (SD) ACT score was 17.4 (5.2). Nearly two-thirds $(62 \%, 47 / 76)$ of these had suboptimal asthma control based on the ACT.

\section{Technology and Device Use}

Participants demonstrated a high level of technology use: $88.8 \%$ (119/134) used a smart phone, 29.9\% (40/134) used health monitoring devices such as a Fitbit, and a small percentage of participants used smart watches $(5.2 \%, 7 / 134)$. Nearly two-thirds $(59.7 \%, 80 / 134)$ used only one form of technology, $26.9 \%$ (36/134) used two forms of technology, and 3.0\% (4/134) used three or more forms of technology. Examples of other specific technology or gadgets used were fitness trackers $(11.9 \%$, $16 / 134)$, tablet computers $(11.9 \%, 16 / 134)$, music players $(3.0 \%$, $4 / 134)$, conventional mobile telephones $(1.4 \%, 2 / 134)$, and electronic books $(1.4 \%, 2 / 134)$. Only 8 participants $(5.9 \%$, $8 / 134$ ) used no "other forms of technology or electronic gadgets". Levels of technology use were similar in those with and without asthma.

\section{Motivation for Wearable Use}

Nearly two-thirds $(61.9 \%, 83 / 134)$ of the total participants indicated that they would be willing to wear a device to monitor their breathing, $7.4 \%$ (10/134) would not, and the remaining $30.5 \%$ (41/134) stated that their willingness depended on specific factors, described later in this section. There were no significant differences in willingness to adopt a wearable device for monitoring breathing between the 40 participants who currently used health monitoring devices and the 94 who did not $(P=.265)$. Participants with asthma were more willing to wear a device to monitor their breathing, compared to those without asthma: $70 \%(54 / 77)$ versus $51 \%(29 / 57), P=.071$.

Regardless of whether or not they were willing to use a wearable, participants were asked to indicate one or more factors that would make them consider using a wearable. These are detailed in Figure 1. Out of all participants, more people who did not have asthma indicated "curiosity" (23\%, 13/57 vs $10 \%$, $7 / 77 ; P=.028)$ or "I would like to track my performance during exercise" $(30 \%, 17 / 57$ vs $10 \%, 8 / 77 ; P=.004)$ as a motivating factor to wear the device than those with asthma.

Females were more likely to use the device to track breathing patterns during stress and meditation compared to men (16\%, $15 / 92$ vs $3 \%, 1 / 39 ; P=.003)$. Females were also more likely to use the device when they get breathless ( $9 \%, 8 / 92$ vs 5\%, 2/39; $P=.002)$ or if they had a known respiratory disease other than asthma compared to men $(8 \%, 7 / 92$ vs $0 \%, 0 / 39 ; P=.031)$.

The ability to track breathing patterns during stress and meditation was a more common rationale for device use in younger than older age groups: $18-39$ (37\%, 7/19), 30-39, (4\%, 1/27), 40-49 (13\%, 2/15), 50-59 (19\%, 5/26), 60-69 (3\%, 1/31), older than $70(0 \%, 0 / 13) ; P=.003$. Curiosity was also a more common rationale for use in younger people: $18-39(42 \%, 8 / 19)$, $30-39$ (22\%, 6/27), 40-49 (7\%, 1/15), 50-59 (4\%, 1/26), 60-69 $(6 \%, 2 / 31)$, older than $70(8 \%, 1 / 13) ; P=.003$.

A larger proportion of the 40 participants who already used a health monitoring device would wear one to monitor their breathing for their asthma or to track patterns during stress (48\%, 19/40 for both), compared to those out of the 94 who did not currently use a device $(29 \%, 27 / 94$ for both; $P=.036)$.

Participants were asked to indicate whether any respiratory illnesses other than asthma were part of their motivation to wear a wearable. Only 8 reported that this was a motivating factor. 
Table 1. Participant demographic information for the wearable survey study, stratified by health status.

\begin{tabular}{|c|c|c|c|}
\hline Characteristic & Total, $\mathrm{n}(\%)(\mathrm{n}=131)^{\mathrm{a}}$ & No asthma, $\mathrm{n}(\%)(\mathrm{n}=55)^{\mathrm{b}}$ & Asthma, $\mathrm{n}(\%)(\mathrm{n}=76)^{\mathrm{c}}$ \\
\hline Gender: Male & $39(29)$ & $22(40)$ & $17(22)$ \\
\hline \multicolumn{4}{|l|}{ Age } \\
\hline $18-29$ & $19(15)$ & $14(25)$ & $5(7)$ \\
\hline $30-39$ & $27(20)$ & $13(23)$ & $14(18)$ \\
\hline $40-49$ & $15(11)$ & $8(15)$ & $7(9)$ \\
\hline $50-59$ & $26(20)$ & $6(11)$ & $20(26)$ \\
\hline $60-69$ & $31(24)$ & $6(11)$ & $25(33)$ \\
\hline $70+$ & $13(10)$ & $8(15)$ & $5(7)$ \\
\hline ACT, mean $(\mathrm{SD})^{\mathrm{d}}$ & - & - & $17.4(5.2)$ \\
\hline \multicolumn{4}{|l|}{ Highest level of education $^{e}$} \\
\hline Secondary school & $21(16)$ & $6(11)$ & $15(20)$ \\
\hline Higher certificate or diploma & $30(23)$ & $9(16)$ & $21(28)$ \\
\hline Bachelor degree or higher & $79(60)$ & $39(71)$ & $40(52)$ \\
\hline Prefer not to say & $1(1)$ & $1(2)$ & $0(0)$ \\
\hline Socioeconomic status: Low SES ${ }^{\mathrm{f}}$ & $25(19)$ & $9(16)$ & $16(32)$ \\
\hline \multicolumn{4}{|l|}{ Employment status ${ }^{\mathrm{g}}$} \\
\hline Employment, full or part time & $80(63)$ & $36(65)$ & $44(61)$ \\
\hline Employment, casual & $12(10)$ & $7(13)$ & $5(7)$ \\
\hline Currently unemployed & $34(27)$ & $11(20)$ & $23(32)$ \\
\hline \multicolumn{4}{|l|}{ Household income (Aus \$) } \\
\hline$\$ 26,000$ & $12(9)$ & $4(7)$ & $8(11)$ \\
\hline$\$ 26,000-\$ 51,999$ & $20(15)$ & $8(15)$ & $12(16)$ \\
\hline$\$ 52,000-\$ 72,799$ & $19(14)$ & $9(16)$ & $10(13)$ \\
\hline$\$ 72,800-\$ 103,999$ & $21(16)$ & $6(11)$ & $15(20)$ \\
\hline$\$ 104,000-\$ 155,999$ & $9(7)$ & $3(5)$ & $6(8)$ \\
\hline$\geq \$ 156,000$ & $23(18)$ & $15(27)$ & $8(11)$ \\
\hline Prefer not to say & $27(21)$ & $10(18)$ & $17(21)$ \\
\hline Language other than English spoken at home & $19(15)$ & $11(20)$ & $8(11)$ \\
\hline
\end{tabular}

${ }^{a} 131 / 134$ participants who completed a survey provided demographic data.

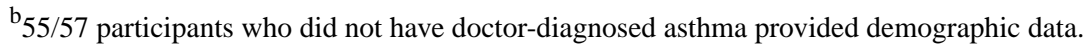

${ }^{\mathrm{c}} 76 / 77$ participants who had doctor-diagnosed asthma provided demographic data.

${ }^{\mathrm{d}}$ A score of $\leq 19$ indicates suboptimal asthma control.

$\mathrm{e}_{1 / 131}$ participants who provided demographic data did not report their education status.

f Socially disadvantaged at patient's home address: "Disadvantaged" Socio-Economic Indexes For Area (SEIFA) quintile <3, "Advantaged" SEIFA quintile: 4-5 [18].

$\mathrm{g}_{2 / 131}$ participants who provided demographic data did not provide employment information; "Currently unemployed" includes unpaid or volunteer work, engagement in home duties, or not being in the labor force.

h 27/131 participants who provided demographic data did not provide household income information. 
Figure 1. User motivation for those willing to use a wearable device, stratified by self-reported, doctor-diagnosed asthma status.

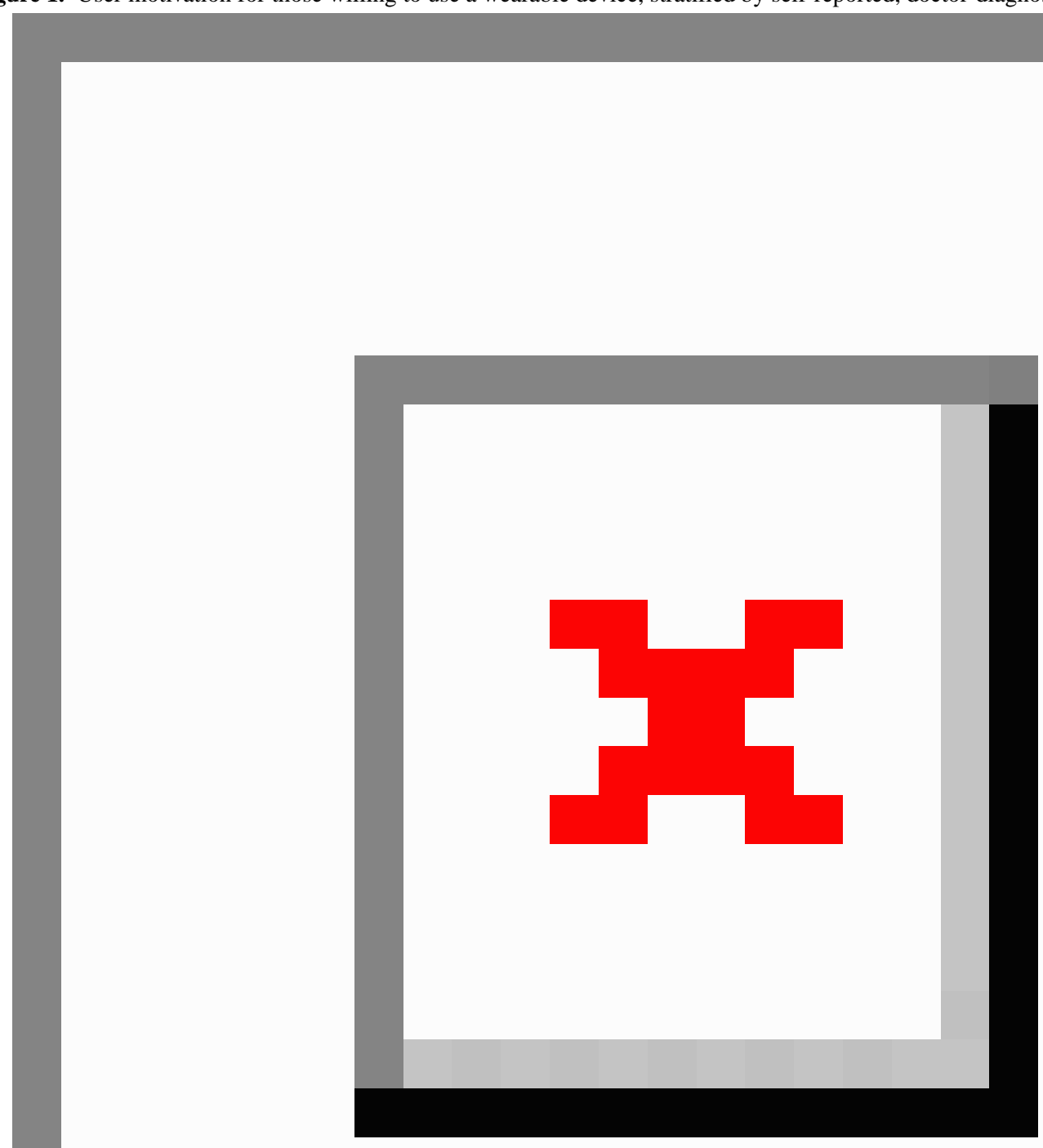

\section{Those Willing to Use a Wearable Device}

When we restricted our analyses to the subgroup of those willing to use a wearable device only $(61.9 \%, 83 / 134)$, the most common motivating factor to wear a device for those without asthma was "curiosity" (59\%, 17/29; $P=.026)$. The most common motivating factor for people with asthma was "I have asthma" $(83 \%, 45 / 54 ; P<.001)$. No significant differences were observed between those with and without asthma in the other provided reasons. Figure 1 shows user motivation across this subgroup, stratified by self-reported, doctor-diagnosed asthma status, with participants able to select multiple responses.

\section{Those Who Would Not Use a Device}

In this subgroup $(6.7 \%, 9 / 134)$, those without asthma stated they would not wear a device because they did not understand why monitoring breathing was important (eg, "I can't see a reason why I would want to monitor my breathing").

The reasons for not using the device in the four participants with asthma were that they felt their asthma was under control (eg, "Asthma is under control," "I don't get bad asthma attacks, just slight, not worth the bother"), or due to travel or cost ("I am overseas at this time," "Such devices are too expensive").

\section{Those Whose Willingness Depended on Specific Factors}

In this subgroup (31.3\%, 42/134), 19 had asthma and 23 did not. The most common motivating factor for wearing a device 
in people with asthma was "I have asthma" (83\%, 14/19; $P<.001)$. No significant factors were found for those without asthma in this subgroup.

\section{Factors Affecting Wearable Use}

The factors affecting wearability mentioned across all participants included design issues and user perception issues. In terms of design, the physical size, location, weight, and bulk of the device were common concerns. Related to these were user perception issues, such as comfort and inhibition of movement, discreetness, and how the device would be fitted to the body. Example of factors provided were "how comfortable and discrete the device is," "how it's worn," "size would it inhibit normal movements and is it 24/7?"

\section{Unappealing Factors}

All participants were asked to select which factors would cause them to consider a wearable device unappealing (Figure 2). Of note, $26 \%(15 / 57)$ of participants without asthma did not see the usefulness of the device, compared to $9 \%(7 / 77)$ of those with asthma $(P=.008)$. More participants without asthma would use a device to monitor breathing only if they were told to by a medical professional compared to those with asthma (39\%, $20 / 49$ vs $17 \%, 13 / 77 ; P=.005)$.

Figure 2. Unappealing factors for wearing a device, stratified by self-reported, doctor-diagnosed asthma.

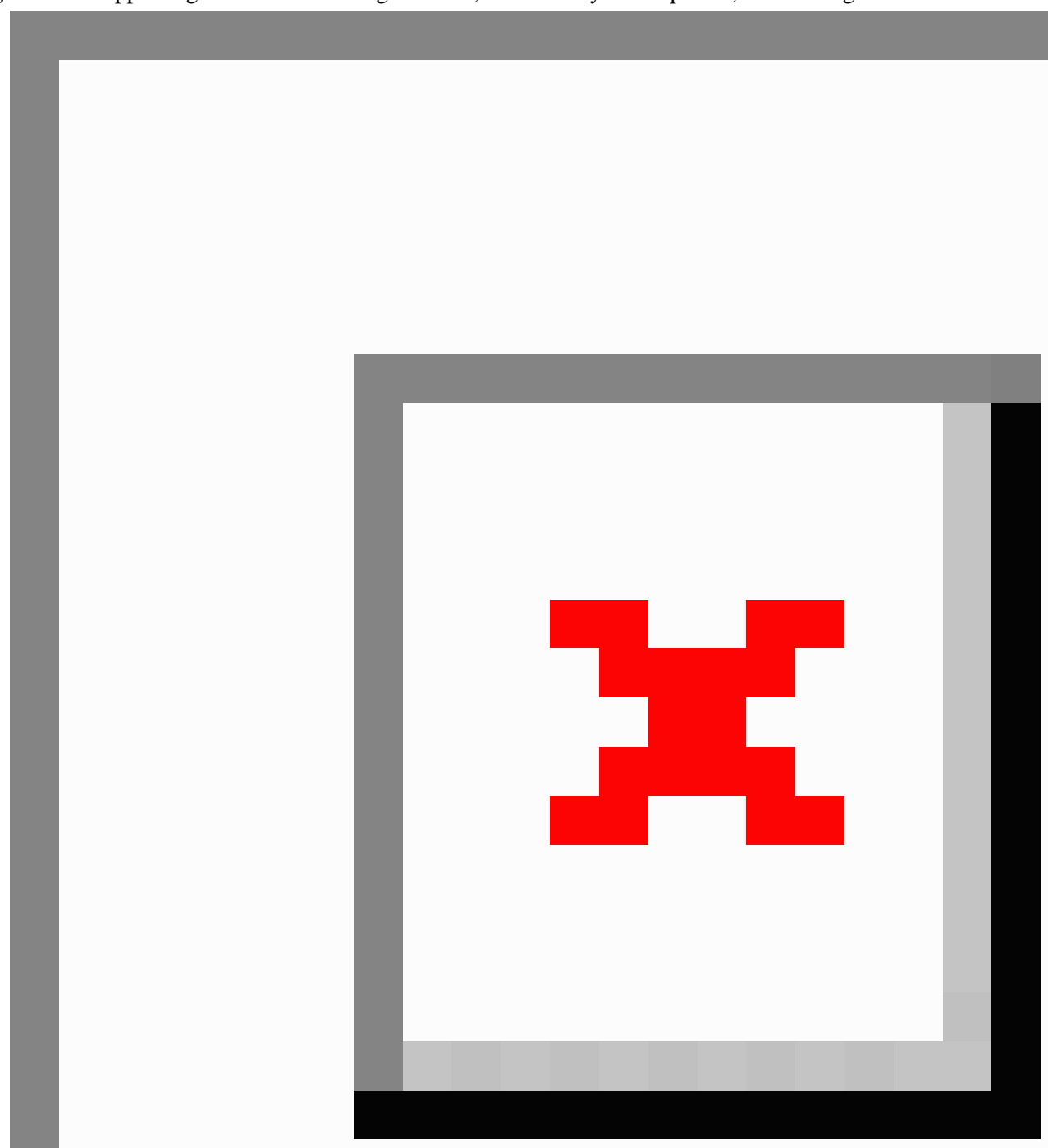




\section{Device-Specific Features}

The device-specific features were themed into five different categories: wearability, cost, power features, display, and data synchronization. All 134 survey participants completed this section. In general, there were no differences between those who were current users of health monitoring devices and those who were not, in preference for form factor, length of usage, cost, display or data storage time preferences, unless otherwise indicated below.

\section{Wearability}

A majority $(94.0 \%, 126 / 134)$ of respondents (with or without asthma) would use a wearable device during the day, night, or both day and night. Most users preferred to wear the device 5 nights/days a week or more (Figure 3). However, more out of those who already used a health monitoring device indicated they would use the device for 5 days or more a week $(83 \%$, $33 / 40)$, compared to those who did not already use a monitoring device $(60 \%, 56 / 94 ; P=.01)$.

Furthermore, those with asthma said they would wear the device more often than those without asthma during both the night and day: $82 \%(63 / 77)$ with asthma versus $46 \%$ (26/57) without asthma would wear the device 5 days or more a week; $P<.001$. Those without asthma were also more likely to wear the device only during training: $26 \%(15 / 57)$ versus $5 \%$ (4/77); $P=.001$. No significance differences were found between health status and form factor for daytime use.

Frequency of daytime and nighttime use was higher in older people. For example, older participants predicted they were more likely to wear the device 5 days a week or more during the night: $18-39$ (37\%, 7/19), 30-39, (59\%, 16/27), 40-49 (53\%, $8 / 15), 50-59(81 \%, 21 / 26), 60-69(74 \%, 23 / 31)$, older than 70 (77\%, 10/13); $P=.026$. Younger age groups were more likely to use the device during exercise than older age groups: $18-39$ (47\%, 9/19), 30-39 (15\%, 4/27), 40-49 (7\%, 1/15), 50-59 (4\%, $1 / 26), 60-69(10 \%, 3 / 31)$, older than $70(8 \%, 1 / 13) ; P=.001$.

There was a clear preference for a wrist band over other formats such as earbuds, and preferences were similar for day versus nighttime use (Figure 4). Men were more likely to wear a chest band during the day $(38 \%, 15 / 39$ vs $20 \%, 18 / 92 ; P=.043)$ compared to women. At night, men were also more likely to wear an ear bud in the ear $(28 \%, 11 / 39$ vs $16 \%, 15 / 92 ; P=.044)$ but less likely to wear a wrist band $(90 \%, 35 / 39$ vs $97 \%, 89 / 92$; $P=.039)$ compared to women.

Figure 3. Total participant preference for how often the device is to be worn, separated by day and night use.

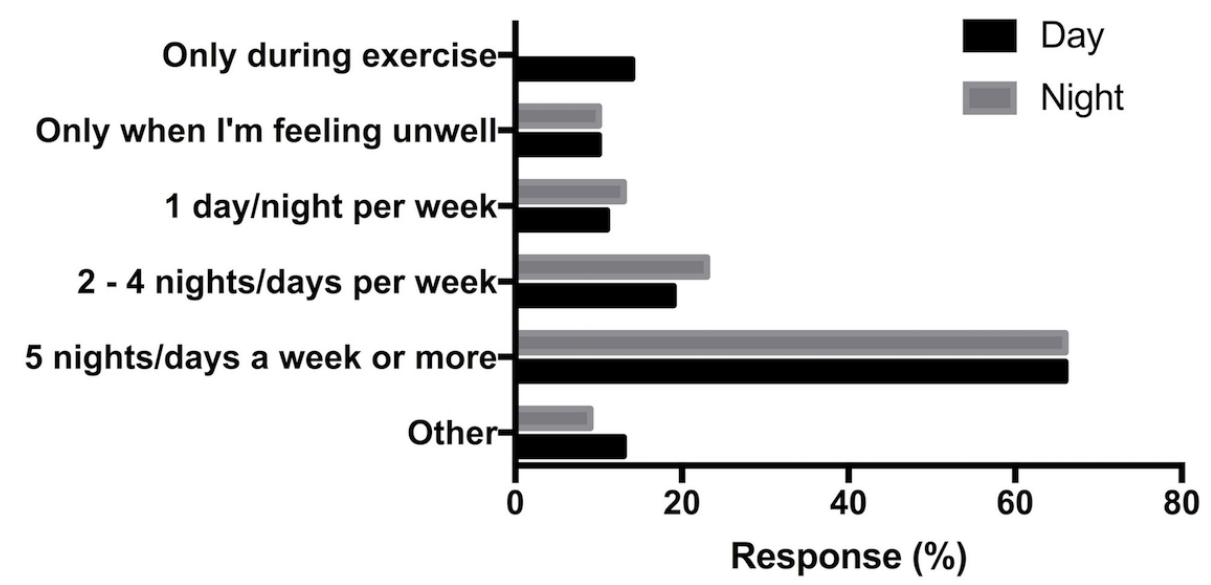

Figure 4. Total participant preference for the form of the device to be worn, separated by day and night use.

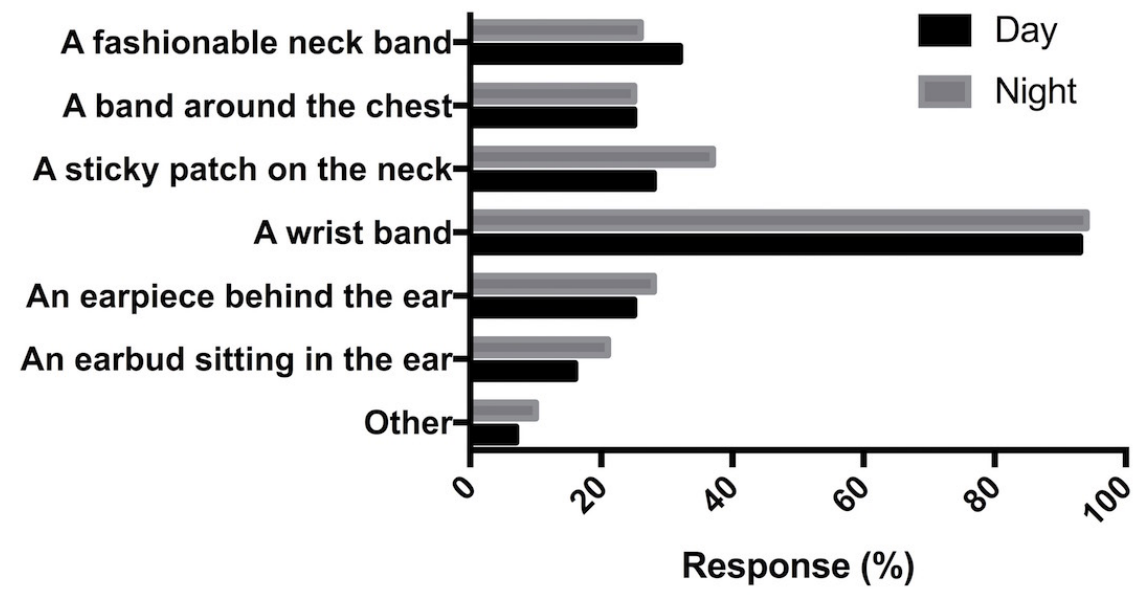




\section{Cost}

Over half $(53.7 \%, 72 / 134)$ of the total participants would be happy to pay up to Aus $\$ 100$ for a wearable respiratory monitor, $20.8 \%$ (28/134) would pay over Aus \$100, and the remaining $25.3 \%$ (34/134) would use it only "if it were free." No statistically significant differences were observed in responses by health status, different household income, age, or gender.

\section{Power Features}

The most popular waiting time for the device to charge was overnight $(45 / 134,33.6 \%)$ as opposed to within 2 hours $(22.3 \%$, $30 / 134)$, 1 hour $(23.1 \%, 31 / 134), 30$ minutes $(11.1 \%, 15 / 134)$, or other $(10.4 \%, 14 / 134)$. Charging time did not appear to be a critical factor in user preferences, with other responses provided as: "As long as it takes. Good if the recharging was no more than 2 hours" or "However long it took to charge." No differences were observed between those with or without asthma.

\section{Display}

Participants selected between the three different displays shown in Figure 5, representing different formats to display current and past breathing data. No preference was found between display type (numerical information, 48/134; bar graph, 39/134; line graph, 47/134). There was no difference in display preference between those with asthma and without asthma, or between different age or gender groups.

Participants indicated that they would like to receive alerts when their breathing was problematic. Alerts were more popular in those with asthma than those without asthma: $79 \%(61 / 77)$ versus $63 \%(36 / 57) ; P=.048$.

\section{Syncing and Data Storage}

The majority of participants $(79.8 \%, 107 / 134)$ reported wanting to sync the device to their phone/tablet. The proportion was higher among those who already use a monitoring device $(93 \%$, $37 / 40)$. Less than half $(45.5 \%, 61 / 134)$ wanted to sync the device with their computer. Those who selected "other" responded with "remote analysis and syncing with my GPs office," "sync with sleep study," or "cloud service." Younger participants were more likely to report wanting to sync their breathing data (number of breaths per minute) with a phone or tablet than older participants: 18-39 (100\%, 19/19), 30-39 (100\%, 27/27), 40-49 (87\%, 13/15), 50-59 (77\%, 20/26), 60-69 (68\%, 21/31), older than $70(54 \%, 7 / 13) ; P=.001$.

The majority of participants reported wanting to save their data for at least 1 week $(58.9 \%, 79 / 134)$.

Figure 5. A display of breathing data by numerical information (left), bar graph (middle), and line graph (right).
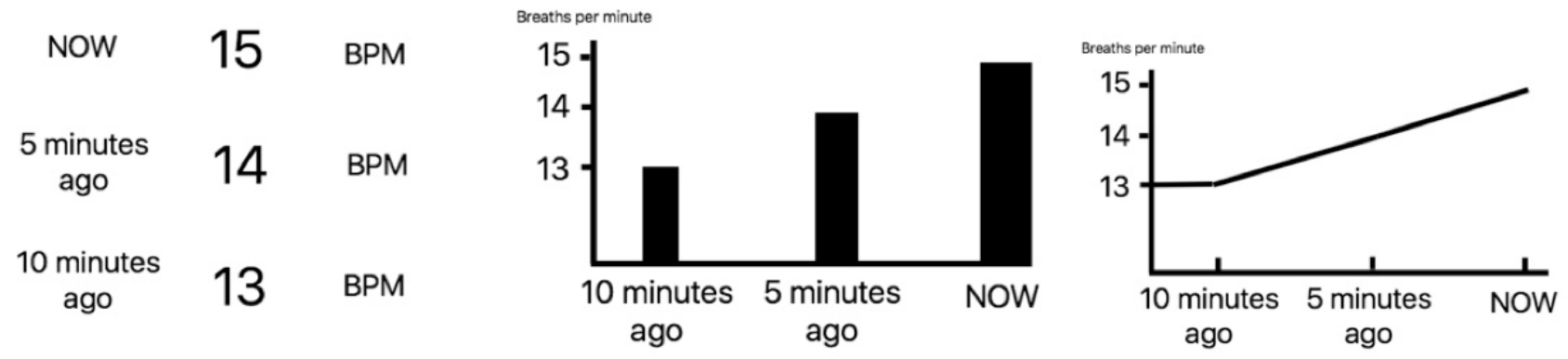

\section{Discussion}

\section{Principal Findings}

In this survey, we identified a number of reasons to adopt new technologies to monitor breathing in participants with or without asthma. In participants without asthma, the main factor that influenced motivation for using a wearable was curiosity. The ability to track breathing patterns during stress or meditation and fitness tracking were motivational factors for younger participants. In asthma, the main motivations for use were "having asthma" and the ability to track breathing patterns during periods of breathlessness. We found that most users were willing to wear the device continuously both day and night and that the most preferred device format was a wrist band, regardless of health status. Other desired features were alerts when breathing is problematic (for both asthma and non-asthma groups), the ability to synchronize data with a phone or tablet, a recharging period of every 24 hours, and cost of $\leq$ Aus $\$ 100$.

\section{Motivation for Wearable Use}

Previous studies have found that perceived value has a significant influence on both potential and actual customers, with perceived value as an important factor influencing the consumer's decision to adopt new products or services [7,11]. One of the most influential factors for people without asthma was curiosity, a factor that in previous research has been thought to increase initial interest and subsequent user engagement [19].

As might be anticipated, motivation for using a wearable device in asthma was different to those without asthma. In people with asthma, there appeared to be a desire to use breathing monitoring to gain greater control over the management of their asthma, particularly during episodes of breathlessness. An episode of extreme breathlessness during a respiratory exacerbation is often extremely frightening to both patients and their family members [20]. Provided that there has been sufficient testing and development of safe and reliable markers, detailed self-tracking breathing metrics could potentially help provide patients with an objective identifier or predictor of such episodes. This is especially important given that self-perception of airway narrowing is known to be poorer during an asthma exacerbation than at other times [20]. For family members, real-time monitoring may allow them to assist in supporting their relative with asthma in identifying symptom worsening and deciding when to seek emergency care, alongside traditional indicators. 
Patients are known to employ a number of strategies to cope with breathlessness episodes, including breathing techniques and reduction of physical exertion [21]. A simple wearable device to measure breathing may provide objective monitoring and feedback during use of breathing techniques, and with the guidance of a health professional, has the potential to support patients to increase their physical activity in a safe manner. A monitor that directly and continuously measures breathing might provide a unique capability for immediate feedback that may not be achieved with currently available devices, such as those measuring wheezing sounds, peak flow, or lung mechanics. There are precedents for monitoring and feedback in asthma, for example, monitoring and feedback of medication use is acceptable and has been shown to increase medication use in adults and children [22,23].

The observed difference in the rationales for using a breathing monitoring device between participants with and without asthma indicates the need to collect separate data on the motivation for use and the utility and feasibility of wearables (for breathing or other purposes), in people with and without (different) health conditions. Conversely, the rationale for choosing not to adopt a wearable device for breathing monitoring was similar between those with and without asthma. The main reason given was a lack of perceived purpose or need for such a device, for example, because asthma was already "under control." Indeed, there is a lack of direct evidence showing that the ambulatory monitoring of breathing patterns over time is useful for asthma. This is despite the disease being characterized by shortness of breath. However, indirect support comes from measurements made using breathing-based lung function tests [24], recent developments in the monitoring of wheeze [25], and data showing breathing patterns predictive of chronic obstructive pulmonary disease exacerbations [5]. The availability of a suitable wearable will enable further work showing utility in asthma management.

\section{User Preference for Device Features}

To the best of our knowledge, this is the first time user preferences for a wearable device aimed at respiratory health monitoring have been investigated. This is important as desired design features often come at a technical cost. The results of this study inform us which features are of high value and which features could be compromised in exchange for technical tradeoffs. Furthermore, acceptance of a new technology may be affected by the perceived risk or inconvenience posed by the device. Previous research suggests that factors such as wearability design, physical size, location, weight, and bulk may negatively impact perceived device value. Costly and complicated recording devices may result in low compliance [10].

There are little data available to suggest what constitutes acceptable levels for these features and for human factors in a breathing monitor wearable. In this study, we found significant user perception issues around comfort and inhibition of movement, discreetness, and where the device fits on the body. Our study also revealed that more than $90 \%$ of participants would wear the device both day and night, and more than $90 \%$ preferred a wrist-worn device. Comfort and frequency of use are likely to interact, with more comfortable devices used for longer.

Most users preferred a wrist band over other formats for site of monitoring; however, this may have been influenced by the type of devices most commonly available on the market at the time. We note that chest bands and ear buds were also identified as next preferred formats for monitoring and may have been selected by participants with existing exposure. Device design choice needs to be made in terms of both user acceptability as well as signal quality. Further study is required to determine the relative feasibility and accuracy in obtaining the breathing signal from these various sites. We did not find significant differences between health groups and their device form preferences.

We found that young participants were more likely to use the device for exercise, but we do not know the reasons why older people were less likely to use such technology for exercise. This could be due to overall lower exercise rates in older people or to less engagement or familiarity with exercise tracking.

Cost can be a barrier to the uptake of monitoring devices, but more than half of our participants would be happy to pay up to Aus \$100 (approximately US \$80) for a wearable that tracks breathing rate. At this price point, such a breathing wearable would be comparable to lower end activity trackers currently on the market and would require a simple design. While creation of a wearable is feasible at this price point, sacrifices in both reliability and comfort may arise. One area of cost reduction could be eliminating a display from the wearable. Any display could be viewed on an external screen such as a mobile phone, while alerts could be processed locally on the device.

Another consideration is device battery life, that is, power consumption must be carefully managed as a small form factor places constraints on battery life [26]. We found device charge time was negotiable, while device use time should be maintained at a minimum of 24 hours. With the size constraint of a wearable, providing this power may be difficult [6]. However, given that the majority of younger participants would like to synchronize data to their mobile phones or tablet, designers may be able to shift data processing functionality to the phone. Furthermore, since participants would like at least a week's worth of data capacity on the device, the requirement for continuous data transmission may also be reduced.

Given the user requirement for data synchronization and data storage, it is recommended that any wearable device should primarily capture and store data. Data transmission to a mobile phone or tablet can take place secondarily by participant demand or when local device storage is full. Any advanced data processing should also take place post transmission.

User security or privacy could potentially be compromised by continuous monitoring [27,28]. We investigated privacy as an unappealing factor in this study but found no observable difference between those willing or unwilling to adopt a breathing monitor. A sample size of 10 for those who would not adopt the device prevented our analyzing a statistically significant difference between the "willingness" groups. 


\section{Limitations}

There are factors limiting the applicability of our findings. The first relates to whether the sample was representative of the population in general. There was a relatively high level of technology use over the population sampled, though only a third of participants were specifically current users of health monitoring devices. Also, $60 \%$ had a university education, a high percentage of respondents were female, and the ages of the study sample were not normally distributed. Although we measured educational level, we did not measure the health literacy of the participants, which may have impacted their responses to the survey. These demographics may not be representative of the general population, and there may have been a selection bias in those who chose to complete the survey (eg, 24\% of those invited from the volunteers database agreed to participate). While we acknowledge there is a potentially high selection bias in those who chose to complete the survey towards those who were already motivated to adopt a wearable, the primary aims of the survey included determining specific user motivation and their preferences for usage and features they wish to have in such a wearable. The population captured was arguably the most appropriate to answer those questions.

Second, while we were able to show differences in the survey responses of those with and without asthma, people without asthma were younger than those with asthma, making it difficult to disentangle the effects of age and disease status. There is some suggestion that older users are more ready to adopt health-related technologies, but the reasons for this require further investigation [29]. More than half of participants with asthma also had suboptimal asthma control.
Third, display preferences were examined in a rudimentary manner in this survey, to determine whether graphical displays were preferred over text. Furthermore, we did not assess in detail whether participants understood how the information was presented, for example, by asking whether they thought the display indicated that their breathing was stable. Once wearable technology is established to measure breathing over time, another study to determine a suitable display of information from the participant's perspective should be explored.

Finally, we did not collect data on whether those who used other health monitoring devices were current or former users, or the reasons for discontinuation of use. Information on how long and why people stay engaged beyond curiosity would have provided major insight into user psychology as well as device development.

\section{Conclusions}

We have explored the motivations for, and the likelihood of, adopting wearable technology for the purpose of breathing monitoring and identified user preferences for key design features. We found participants were motivated to adopt a wearable breathing monitor regardless of health status, yet there were distinctly different rationales for use between those with and without asthma. There is a clear need to identify the benefits of monitoring breathing in health and asthma. Next steps will require the development and testing of reliable breathing metrics or indicators that can be safely used by people with asthma for monitoring breathing over time or that assist in the identification of symptom worsening and asthma exacerbations. These findings will help inform the design of a user-acceptable wearable device that will facilitate its eventual uptake in both healthy and asthma populations.

\section{Acknowledgments}

The authors acknowledge the role of the Google Impact Challenge Grant, which provided seed funding for the greater study. We also acknowledge the role of Michele Goldman of Asthma Australia in co-development of the proposal leading to the seed funding.

\section{Conflicts of Interest}

JBP was partially funded by an Australian Postgraduate Award and a philanthropic Google Grant, of which PMY and ET were primary investigators in collaboration with Asthma Australia. In the last 3 years, the Woolcock Institute of Medical Research has received independent research funding from AstraZeneca and GlaxoSmithKline for asthma research carried out by JMF.

\section{Multimedia Appendix 1}

Online questionnaire.

[PDF File (Adobe PDF File), 326KB-Multimedia Appendix 1]

\section{References}

1. GINA. Global Initiative for Asthma. Global Strategy for Asthma Management and Prevention (2015 update) URL: http:/ /ginasthma.org/wp-content/uploads/2016/01/GINA_Report_2015_Aug11-1.pdf [accessed 2016-11-06] [WebCite Cache ID 6lpKrwOja]

2. Frey U, Suki B. Complexity of chronic asthma and chronic obstructive pulmonary disease: implications for risk assessment, and disease progression and control. Lancet 2008 Sep 20;372(9643):1088-1099 [FREE Full text] [doi:

10.1016/S0140-6736(08)61450-6] [Medline: 18805337]

3. Thamrin C, Taylor DR, Jones SL, Suki B, Frey U. Variability of lung function predicts loss of asthma control following withdrawal of inhaled corticosteroid treatment. Thorax 2010 May;65(5):403-408. [doi: 10.1136/thx.2009.129668] [Medline: 20435861] 
4. Thamrin C, Zindel J, Nydegger R, Reddel HK, Chanez P, Wenzel SE, et al. Predicting future risk of asthma exacerbations using individual conditional probabilities. J Allergy Clin Immunol 2011 Jun;127(6):1494-1502.e3. [doi: 10.1016/j.jaci.2011.01.018] [Medline: 21333347]

5. Yañez AM, Guerrero D, Pérez de Alejo R, Garcia-Rio F, Alvarez-Sala JL, Calle-Rubio M, et al. Monitoring breathing rate at home allows early identification of COPD exacerbations. Chest 2012 Dec;142(6):1524-1529. [doi: 10.1378/chest.11-2728] [Medline: 22797131]

6. Williamson J, Liu Q, Lu F, Mohrman W, Li K, Dick R, et al. Data sensing and analysis: Challenges for wearables. In: IEEE. 2015 Presented at: Design Automation Conference (ASP-DAC), 2015 20th Asia and South Pacific; Jan. 19-22, 2015; Chiba, Japan. [doi: 10.1109/ASPDAC.2015.7058994]

7. Motti V, Caine K. Human factors considerations in the design of wearable devices. Proceedings of the Human Factors and Ergonomics Society 2014;58(1):1820-1824.

8. Bergmann JH, McGregor AH. Body-worn sensor design: what do patients and clinicians want? Ann Biomed Eng 2011 Sep;39(9):2299-2312. [doi: 10.1007/s10439-011-0339-9] [Medline: 21674260]

9. Knight J, Deen-Williams D, Arvantis T, Baber C, Sotiriou S, Anastopoulou S, et al. Assessing the Wearability of Wearable Computers. 2006 Presented at: 10th IEEE International Symposium on Wearable Computers; Oct. 11-14, 2006; Montreux, Switzerland p. 75-82. [doi: 10.1109/ISWC.2006.286347]

10. Yang H, Yu J, Zo H, Choi M. User acceptance of wearable devices: An extended perspective of perceived value. Telematics and Informatics 2016 May;33(2):256-269. [doi: 10.1016/j.tele.2015.08.007]

11. Buenaflor C, Kim H. Six human factors to acceptability of wearable computers. International Journal of Multimedia and Ubiquitous Engineering 2013;8(3):103-114.

12. Rossi S, Pessione M, Torre L. A low power bioimpedance module for wearable systems. Procedia Engineering 2014;87:1274-1277. [doi: 10.1016/j.proeng.2014.11.419]

13. Charlton PH, Bonnici T, Tarassenko L, Clifton DA, Beale R, Watkinson PJ. An assessment of algorithms to estimate respiratory rate from the electrocardiogram and photoplethysmogram. Physiol Meas 2016 Apr;37(4):610-626 [FREE Full text] [doi: 10.1088/0967-3334/37/4/610] [Medline: 27027672]

14. Rahman T, Adam A, Zhang M, Cherry E, Zhou B, Peng H, et al. BodyBeat: a mobile system for sensing non-speech body sounds. In: MobiSys. 2014 Presented at: 12th Annual International Conference on Mobile Systems, Applications, and Services; June 16-19, 2014; Bretton Woods, NH, USA p. 2-13. [doi: 10.1145/2594368.2594386]

15. Ledger D, McCaffrey D. Inside wearables: How the science of human behavior change offers the secret to long-term engagement. Endeavour Partners 2014;200(93):1.

16. Nathan R. Development of the Asthma Control Test: A survey for assessing asthma control. Journal of Allergy and Clinical Immunology 2004;113(1):59-65. [doi: 10.1016/j.jaci.2003.09.008]

17. Al Moamary MS, Al Kordi AG, Al Ghobain MO, Tamim H. Utilization and responsiveness of the asthma control test (ACT) at the initiation of therapy for patients with asthma: A randomized controlled trial. BMC Pulmonary Medicine 2012;12(1):14. [doi: 10.1186/1471-2466-12-14]

18. Australian Bureau of Statistics. Socio-economic indexes for areas (Technical paper). 2011. Census of Population and Housing URL: http://www.abs.gov.au/ausstats/abs@.nsf/Lookup/2033.0.55.001main+features52011 [accessed 2017-06-22] [WebCite Cache ID 6rPlyQ5PJ]

19. Arnone M, Small RV, Chauncey SA, McKenna HP. Curiosity, interest and engagement in technology-pervasive learning environments: a new research agenda. Education Tech Research Dev 2011 Feb 27;59(2):181-198. [doi: 10.1007/s11423-011-9190-9]

20. Salome CM, Leuppi JD, Freed R, Marks GB. Perception of airway narrowing during reduction of inhaled corticosteroids and asthma exacerbation. Thorax 2003 Dec;58(12):1042-1047 [FREE Full text] [Medline: 14645970]

21. Simon S. I Can Breathe Again! Patients' Self-Management Strategies for Episodic Breathlessness in Advanced Disease, Derived From Qualitative Interviews. Journal of Pain and Symptom Management 2016;52:228-234. [doi: 10.1016/j.jpainsymman.2016.02.016]

22. Foster J, Usherwood T, Smith L, Sawyer S, Xuan W, Rand C, et al. Inhaler reminders improve adherence with controller treatment in primary care patients with asthma. Journal of Allergy and Clinical Immunology 2014;134(6):1260-1268. [doi: 10.1016/j.jaci.2014.05.041]

23. Chan A, Stewart AW, Harrison J, Camargo CA, Black PN, Mitchell EA. The effect of an electronic monitoring device with audiovisual reminder function on adherence to inhaled corticosteroids and school attendance in children with asthma: a randomised controlled trial. The Lancet Respiratory Medicine 2015 Mar;3(3):210-219. [doi: 10.1016/S2213-2600(15)00008-9]

24. Gulotta C, Suki B, Brusasco V, Pellegrino R, Gobbi A, Pedotti A, et al. Monitoring the temporal changes of respiratory resistance: a novel test for the management of asthma. Am J Respir Crit Care Med 2012 Jun 15;185(12):1330-1331 [FREE Full text] [doi: 10.1164/ajrccm.185.12.1330] [Medline: 22707741]

25. Bentur L, Beck R, Berkowitz D, Hasanin J, Berger I, Elias N, et al. Adenosine bronchial provocation with computerized wheeze detection in young infants with prolonged cough: correlation with long-term follow-up. CHEST Journal 2004;126(4):1060-1065. [doi: 10.1378/chest.126.4.1060] [Medline: 15486364] 
26. Klingeberg T, Schilling M. Mobile wearable device for long term monitoring of vital signs. Computer Methods and Programs in Biomedicine 2012;106(2):89-96. [doi: 10.1016/j.cmpb.2011.12.009]

27. Page T. Privacy Issues Surrounding Wearable Technology. i-Manager's Journal on Information Technology 2015;4(4):1-16.

28. Di Pietro R, Mancini LV. Security and privacy issues of handheld and wearable wireless devices. Commun ACM 2003 Sep 01;46(9):74-79. [doi: 10.1145/903893.903897]

29. Arning K, Ziefle M. Different perspectives on technology acceptance: The role of technology type and age. In: Lecture Notes in Computer Science (including subseries Lecture Notes in Artificial Intelligence and Lecture Notes in Bioinformatics): Springer; 2009 Presented at: Symposium of the Austrian HCI and Usability Engineering Group; 2009; Austria. [doi: 10.1007/978-3-642-10308-7 2]

\section{Abbreviations}

ACT: Asthma Control Test

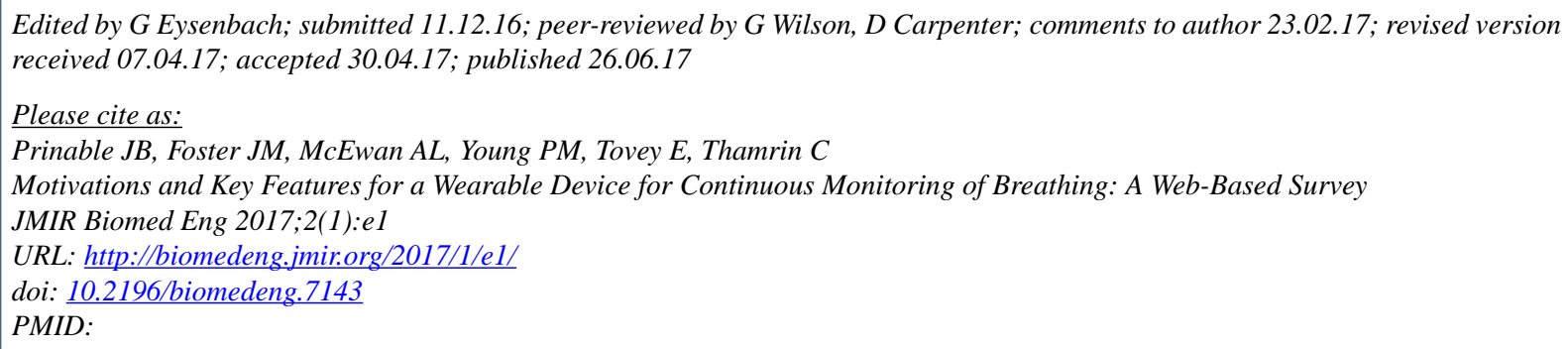

CJoseph Barry Prinable, Juliet M Foster, Alistair L McEwan, Paul M Young, Euan Tovey, Cindy Thamrin. Originally published in JMIR Biomedical Engineering (http://biomedeng.jmir.org), 26.06.2017. This is an open-access article distributed under the terms of the Creative Commons Attribution License (https://creativecommons.org/licenses/by/4.0/), which permits unrestricted use, distribution, and reproduction in any medium, provided the original work, first published in JMIR Biomedical Engineering, is properly cited. The complete bibliographic information, a link to the original publication on http://biomedeng.jmir.org/, as well as this copyright and license information must be included. 